\section{Rift Valley Fever and Crimean-Congo Hemorrhagic Fever Viruses in Ruminants, Jordan}

\author{
Mohammad M. Obaidat, James C. Graziano, \\ Maria Morales-Betoulle, Shelley M. Brown, \\ Cheng-Feng Chiang, John D. Klena
}

Author affiliations: Jordan University of Science and Technology, Irbid, Jordan (M.M. Obaidat); Centers for Disease Control and Prevention, Atlanta, Georgia, USA (J.C. Graziano, M. Morales-Betoulle, S.M. Brown, C.-F. Chiang, J.D. Klena)

DOI: https://doi.org/10.3201/eid2702.203713

The epidemiology of Rift Valley fever virus (RVFV) and Crimean-Congo hemorrhagic fever virus (CCHFV) in Jordan is unknown. Our investigation showed $3 \%$ of 989 tested dairy cattle, sheep, and goats were RVFV seropositive and $14 \%$ were CCHFV seropositive. Ongoing surveillance is needed to assess risk to humans and protect public health.

$\mathrm{R}$ ift Valley fever (RVF) virus (RVFV) and CrimeanCongo hemorrhagic fever (CCHF) virus (CCHFV) are zoonotic arboviruses. RVFV has been causing sporadic outbreaks in East, West, and southern Africa; the Indian Ocean region; and the Arabian Peninsula (Saudi Arabia and Yemen) (1). Although Jordan is considered an at-risk country, the disease has not been reported in Jordan (2). Meanwhile, no seroprevalence studies for CCHFV in human or animals have been conducted in Jordan despite the endemicity of CCHF in neighboring countries (https://www.cdc.gov/vhf/ crimean-congo/outbreaks/distribution-map.html), the presence of a necessary tick vector (Hyalomma sp.) (http://www.who.int/csr/disease/crimean_congo$\mathrm{HF}$ ), and the classification of Jordan as an at-risk country (3). Accordingly, we aimed to determine whether livestock populations across Jordan have been exposed to CCHFV and RVFV (Appendix, https://wwwnc. cdc.gov/EID/article/27/2/20-3713-App1.pdf). Jordan University of Science and Technology Animal Care and Use Committee approved the study.

Using EpiTool (https://epitools.ausvet.com.au), we determined that a minimum of 665 samples were required based on an assumed prevalence of $0.5 \%$ and a $95 \%$ CI. We tested 989 serum samples from 109 farms (31 dairy cow farms, 44 sheep farms, and 20 goat farms, as well as 14 mixed sheep and goat farms) that were randomly selected from different regions of Jordan during 2015-2016. Serum samples were shipped to the US Centers for Disease Control and Prevention (Atlanta, Georgia USA) for laboratory testing by indirect ELISA (Appendix).

Overall seroprevalence was $14 \%$ for CCHFV and $3 \%$ for RVFV. The greatest differences in seroprevalence were among sheep, $16.7 \%$ (85/509) for CCHFV and $4.5 \%(23 / 509)$ for RVFV, followed by a similar difference for goats, $14.7 \%$ (48/327) for CCHFV and $0.6 \%$ (2/327) for RVFV (Table). CCHFV and RVFV seroprevalence did not differ in cows at $\approx 1 \%$ (4/152 for CCHF and 2/152 for RVF) (Table).

The provinces that had the highest respective seroprevalence for CCHFV or RVFV did not coincide (Figure). The highest CCHFV seroprevalence was found in the northwest and the highest RVFV seroprevalence in the provinces along the central western border area with Israel (Figure). In total, 29 farms had seropositivity for CCHFV: 19 sheep farms (10 in Irbid, 5 in Tafela, 2 in Jarash, 1 in Ma'an, and 1 in Mafraq), 5 mixed sheep and goat farms ( 1 in each of Irbid, Jarash, Ajloun,

\begin{tabular}{lcccccccccccccccc}
\hline \multicolumn{1}{l}{ Table. Seroprevalence of CCHFV and RVFV by location and animal species, Jordan, 2015-2016* } \\
\hline
\end{tabular}

${ }^{*}$ CCHFV, Crimean-Congo hemorrhagic fever virus; RVFV, Rift Valley fever virus. 

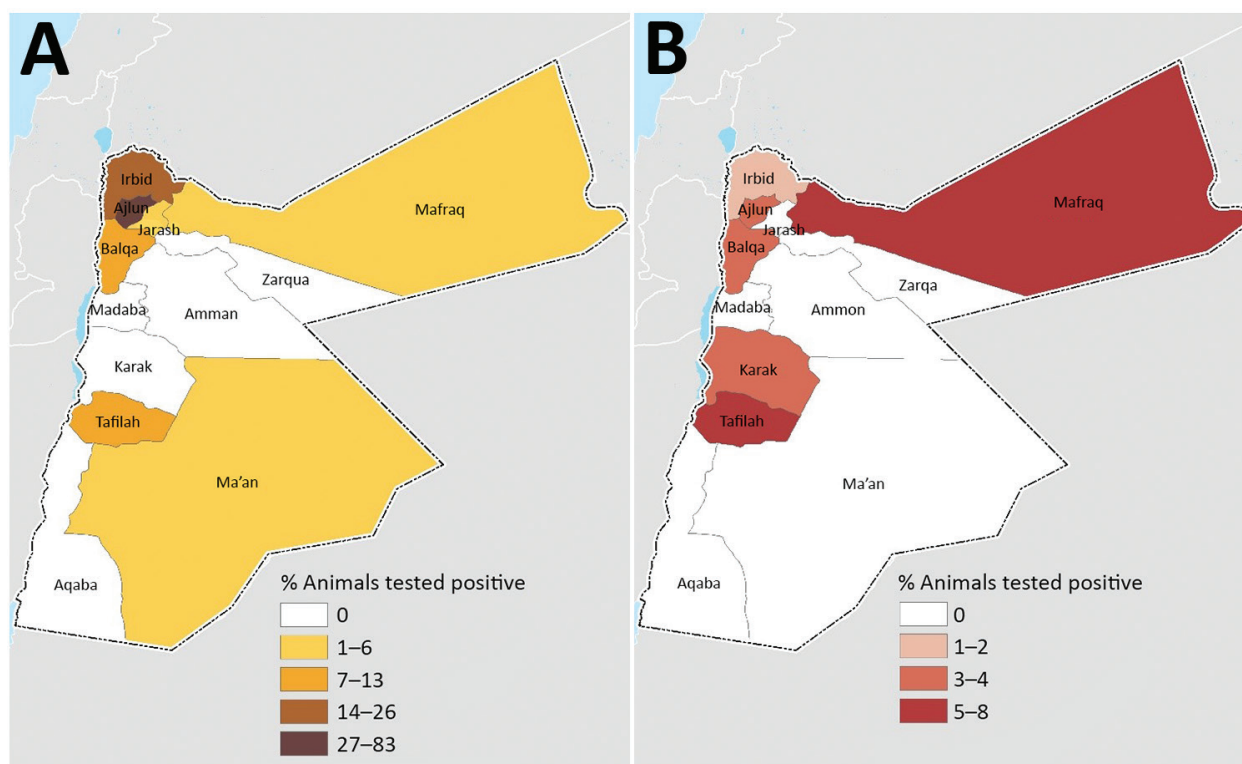

Figure. Seroprevalence of Crimean-Congo hemorrhagic fever (A) and Rift Valley fever (B) in ruminants, by province, Jordan, 2015-2016.

Mafraq, and Balqa), 3 goat farms (all in Jarash), and 2 dairy cow farms in Irbid. Ten farms had animals seropositive for RVFV: 5 sheep farms ( 2 in Tafelah, 2 in Irbid, and 1 in Mafraq), 3 mixed sheep and goat farms ( 1 in each of Ajloun, Mafraq, and Balqa), 1 goat farm in Karak, and 1 dairy-cow farm in Zarqa.

This study reports RVFV seropositivity in Jordan's ruminant population without any previously reported animal cases. Observing seropositive animals without disease, however, is not unique; $22 \%$ of the small ruminant population in Mayotte were seropositive (4) without any documented human or animal clinical cases. Similarly, South Africa reported high proportion of seropositive ruminants in the absence of a reported outbreak (5). In addition, IgG seroprevalence of $6.5 \%$ was detected in sheep and goats in southern Gabon without a reported outbreak (6).

In Jordan, small ruminants are short day breeders; June-September are breeding months. After a $\approx 5$-month gestation period, lambing occurs during November-February, which places gestation and lambing periods during the rainy months in Jordan. The shift of RVF from enzootic to epizootic or epidemic cycle typically follows extended periods of heavy rainfall (7). Because rainy season and gestation periods overlap, RVFV spread poses a potential high risk for abortions and neonatal death in Jordan.

In light of the regional distribution and general expansion of RVFV and CCHFV into newly identified areas, it is not surprising that animals in Jordan tested seropositive to either virus. This finding is consistent with recent studies that reported other mosquitoborne viruses in Jordan, such as West Nile (8) and dengue viruses (9), and tickborne viruses such as Coxiella burnetii (10).

The findings of seropositive animals for CCHFV and RVFV in different regions of Jordan call for implementing an early warning contingency plan. Such a plan would include training field veterinary officers, developing strong epidemiologic capabilities, sustaining active disease surveillance, and enhancing laboratory diagnostic capabilities. On the basis of our identification of the subprovinces with the highest seroprevalence, small ruminant sentinel herds should be monitored for IgG and IgM to these viruses in conjunction with seasonal weather, particularly before and during the rainy months. Despite CCHF virulence in humans and the potential public health impact because of severe outbreaks, the virus is not pathogenic for the amplifying hosts (i.e., ruminants). Thus, farmers and veterinarians are at higher risk for infection compared with the general population. Future studies should be conducted to determine the prevalence and potential incident cases of CCHF and RVF in Jordan's human and animal populations. Ongoing surveillance will inform contemporaneous risk assessments and enable development of effective public health messaging for identified risk groups.

\section{Acknowledgments}

We thank Alaa E. Bani Salman and Amany Rashaideh for their assistance. We thank the farmers and herdsmen for allowing the sampling of their herds and flocks.

Part of this study was supported by Deanship of Research at Jordan University of Science and Technology (grant no. 20160280). 


\section{About the Author}

Dr. Obaidat is associate professor at the faculty of veterinary medicine at Jordan University of Science and Technology. His research interest includes the epidemiology of zoonotic diseases in Jordan.

\section{References}

1. Samy AM, Peterson AT, Hall M. Phylogeography of Rift Valley fever virus in Africa and the Arabian Peninsula. PLoS Negl Trop Dis. 2017;11:e0005226. https://doi.org/10.1371/ journal.pntd.0005226

2. EFSA Panel on Animal Health and Welfare (AHAW). Scientific opinion on Rift Valley fever. EFSA J. 2013;11:3180. https://doi.org/10.2903/j.efsa.2013.3180

3. Spengler JR, Bente DA, Bray M, Burt F, Hewson R, Korukluoglu G, et al. Second international conference on Crimean-Congo hemorrhagic fever. Antiviral Res. 2018;150:137-47. https://doi.org/10.1016/ j.antiviral.2017.11.019

4. Lernout $\mathrm{T}$, Cardinale $\mathrm{E}$, Jego $\mathrm{M}$, Desprès $\mathrm{P}$, Collet $\mathrm{L}$, Zumbo B, et al. Rift Valley fever in humans and animals in Mayotte, an endemic situation? PLoS One. 2013;8:e74192. https://doi.org/10.1371/journal.pone.0074192

5. van den Bergh C, Venter EH, Swanepoel R, Thompson PN. High seroconversion rate to Rift Valley fever virus in cattle and goats in far northern KwaZulu-Natal, South Africa, in the absence of reported outbreaks. PLoS Negl Trop Dis. 2019;13:e0007296. https:/ / doi.org/10.1371/journal. pntd.0007296

6. Maganga GD, Abessolo Ndong AL, Mikala Okouyi CS, Makiala Mandanda S, N'Dilimabaka N, Pinto A, et al. Serological evidence for the circulation of Rift Valley fever virus in domestic small ruminants in southern Gabon. Vector Borne Zoonotic Dis. 2017;17:443-6. https:/ / doi.org/10.1089/ vbz.2016.2065

7. Anyamba A, Linthicum KJ, Small J, Britch SC, Pak E, de La Rocque S, et al. Prediction, assessment of the Rift Valley fever activity in east and southern Africa 2006-2008 and possible vector control strategies. Am J Trop Med Hyg. 2010;83:Suppl 43-51. https:// doi.org/10.4269/ ajtmh.2010.09-0289

8. Obaidat MM, Stringer AP, Roess AA. Seroprevalence, risk factors and spatial distribution of West Nile virus in Jordan. Trans R Soc Trop Med Hyg. 2019;113:24-30. https://doi.org/10.1093/trstmh/try111

9. Obaidat MM, Roess AA. First report on seroprevalence and risk factors of dengue virus in Jordan. Trans R Soc Trop Med Hyg. 2018;112:279-84. https://doi.org/10.1093/trstmh/ try055

10. Obaidat MM, Kersh GJ. Prevalence and risk factors of Coxiella burnetii antibodies in bulk milk from cattle, sheep, and goats in Jordan. J Food Prot. 2017;80:561-6. https:/ / doi.org/10.4315/0362-028X.JFP-16-377

Address for correspondence: Mohammad M. Obaidat, Department of Veterinary Pathology and Public Health, Faculty of Veterinary Medicine, Jordan University of Science and Technology, Irbid, Jordan; email: mmobaidat@just.edu.jo; and John D. Klena, Centers for Disease Control and Prevention, 1600 Clifton Rd NE, Mailstop H18-SSB, Atlanta, GA 30329-4027, USA; email: irc4@cdc.gov

\section{Genomic Diversity of Burkholderia pseudomallei Isolates, Colombia}

\author{
Carolina Duarte, Franco Montufar, Jaime Moreno, \\ Dora Sánchez, Jose Yesid Rodríguez, \\ Alfredo G. Torres, Soraya Morales, Adriana Bautista, \\ Mónica G. Huertas, Julia N. Myers, \\ Christopher A. Gulvik, Mindy G. Elrod, \\ David D. Blaney, Jay E. Gee
}

\begin{abstract}
Author affiliations: Instituto Nacional de Salud, Bogotá, Colombia (C. Duarte, J. Moreno, D. Sanchez, A. Bautista); Clínica León XIII Universidad de Antioquia, Medellín, Colombia (F. Montufar); Centro de Investigaciones Microbiológicas del Cesar, Valledupar, Colombia (J.Y. Rodriguez); University of Texas Medical Branch, Galveston, Texas, USA (A.G. Torres, J.N. Myers); Universidad de Santander, Valledupar, Colombia (S. Morales); Universidad El Bosque, Bogotá, Colombia (M.G. Huertas); Centers for Disease Control and Prevention, Atlanta, Georgia, USA (C.A. Gulvik, M.G. Elrod, D.D. Blaney, J.E. Gee)
\end{abstract}

DOI: https://doi.org/10.3201/eid2702.202824

We report an analysis of the genomic diversity of isolates of Burkholderia pseudomallei, the cause of melioidosis, recovered in Colombia from routine surveillance during 2016-2017. B. pseudomallei appears genetically diverse, suggesting it is well established and has spread across the region.

M elioidosis is caused by the environmental bacterium Burkholderia pseudomallei. Infections are acquired by direct contact with the pathogen, most commonly through traumatic inoculation with contaminated soil or water but also by ingestion or inhalation. Symptoms are nonspecific and can include pneumonia, skin lesions, abscess formation, and sepsis (1).

In Latin America, melioidosis is believed to be underdiagnosed because of the absence of reliable surveillance and the lack of available diagnostic tools and methods (2). Colombia has previously reported cases as sporadic, isolated events in a few geographic areas $(2,3)$. The aim of this study was to genetically characterize isolates of $B$. pseudomallei recovered from clinical specimens in different departments of Colombia (4). (A department in Colombia is a geographic unit composed of municipalities led by a governor.) The goal was to better understand genetic relationships among the isolates from Colombia, as well as their relationships to isolates from other tropical and subtropical regions of the Americas. The study was internally reviewed at the US Centers for Disease 